\title{
Temporomandibular joint reconstruction with costochondral graft: case series study
}

\author{
Moon Gi Choi \\ Department of Oral and Maxillofacial Surgery, College of Dentistry, Wonkwang University, Iksan, Korea
}

\begin{abstract}
J Korean Assoc Oral Maxillofac Surg 2021;47:128-134)
Various techniques have been used to reconstruct the temporomandibular joints, including autogenous transplants and alloplastic implants. Among autogenous grafts, costochondral grafts have mainly been used. A costochondral graft has many advantages over other autogenous grafts and alloplastic implants. Harvest is easy and has minimal impact on patients. The graft can bear functional load well and biocompatibility is excellent. A costochondral graft obviates foreign body reactions and further surgery for revision of alloplastic replacements if the graft takes well. Although long-term prognosis remains unclear, it appears that for autogenous condylar reconstruction, costochondral grafts can be used with few complications and acceptable results. This article describes cases and discusses surgical techniques and considerations related to costochondral grafts.
\end{abstract}

Key words: Costochondral graft, Temporomandibular joint, Autogenous graft

[paper submitted 2020. 9. 8 / revised 2020. 10. 26 / accepted 2020. 11. 16]

\section{Introduction}

The indications for reconstruction of the temporomandibular joint (TMJ) include ankylosis, end-stage osteoarthritis, rheumatoid arthritis, tumor of condyle, traumatic deficit, and congenital pathology ${ }^{1}$.

Various methods have been used to reconstruct the TMJ including autogenous transplants and alloplastic implants. Among the autogenous grafts, fibular, metatarsal, sternoclavicular, iliac, and costochondral grafts have been used. Among the alloplastic grafts, total TMJ system is most widely used ${ }^{1}$.

Costochondral transplantation has many advantages over other autogenous transplants. Harvesting is easy and has minimal impact on patients. The transplant bears functional loading well and has excellent biocompatibility. A costochon-

\section{Moon Gi Choi \\ Department of Oral and Maxillofacial Surgery, College of Dentistry, Wonkwang University, 460 Iksan-daero, Iksan 54538, Korea \\ TEL: +82-63-859-2921 \\ E-mail: omschoi@wku.ac.kr \\ ORCID: https://orcid.org/0000-0003-3502-7652}

(C) This is an open-access article distributed under the terms of the Creative Commons Attribution Non-Commercial License (http://creativecommons.org/ licenses/by-nc/4.0/), which permits unrestricted non-commercial use, distribution, and reproduction in any medium, provided the original work is properly cited.

Copyright (C) 2021 The Korean Association of Oral and Maxillofacial Surgeons. All rights reserved. dral graft in children has the potential for growth. Despite these advantages, there are many potential problems. Costochondral grafts can be fractured and ankylosis can occur after transplantation. In addition, such a graft can show various growth behaviors. Although donor site morbidity is minimal, the wound can be bothersome to patients ${ }^{2,3}$.

\section{Cases Report}

Here, 4 patients with 5 operated joints were retrospectively reviewed. All patients were adults and received a costochondral graft at Wonkwang Dental Hospital due to diverse causes from 2005 to 2020. Issues regarding function, pain, long-term results, and adverse outcomes are presented.

\section{Surgical techniques}

The surgical field was opened through preauricular and/or submandibular incision. To obtain a definite surgical field, we used both preauricular and submandibular approaches in the first few cases. However, as our experience increased, only one of the approaches was used.

Costochondral graft was taken from the right chest at the 5th, 6th, and 7th rib bones through inframammary incision. The reason for collecting graft from the right side was to dis- 
tinguish postoperative pain from cardiac pain. If two grafts were harvested for bicondylar reconstruction, tissue was collected from separate rib bones to prevent sinking of the tho-

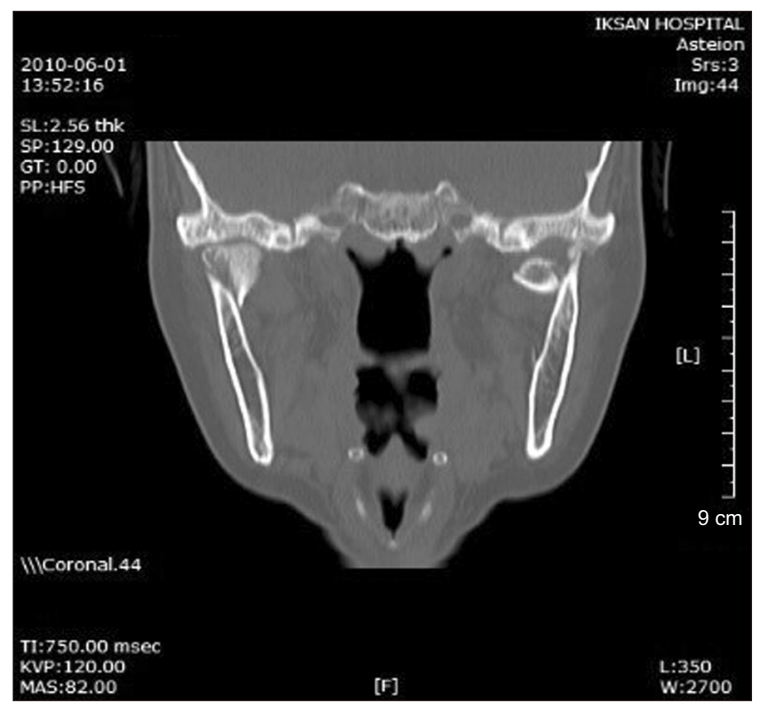

Fig. 1. On computed tomography, bilateral intracapsular condylar fractures were observed.

Moon Gi Choi: Temporomandibular joint reconstruction with costochondral graft: case series study. J Korean Assoc Oral Maxillofac Surg 2021 racic cage, leaving intact rib bone between them. Such a graft was generally about $5 \mathrm{~cm}$ in length, and the cartilage portion was trimmed to $2-3 \mathrm{~mm}$ in thickness.

Only if the TMJ disc was degenerated was it removed. Graft was pushed upward to contact the disc or articular surface loosely in centric occlusion and fixed to the ramus with a washer technique using a miniplate and bicortical screws. The minimum number of screws was three, and the graft had to be fixed with no change in occlusion. Condylar movement and occlusion were measured at mouth closed and open positions. The mouth opening in the operation room had to be $3.0-3.5 \mathrm{~cm}$ in length after reconstruction.

As mouth opening increased, the end portion of the costochondral graft might move along the articular surface and eminence. If mouth opening was not improved, unilateral or bilateral condylectomy was performed to secure the ideal opening.

In our first cases, intermaxillary fixation for bony consolidation was used for three weeks. As our experience increased, we ceased use of intermaxillary fixation. Instead, active opening exercises were scheduled for several months.
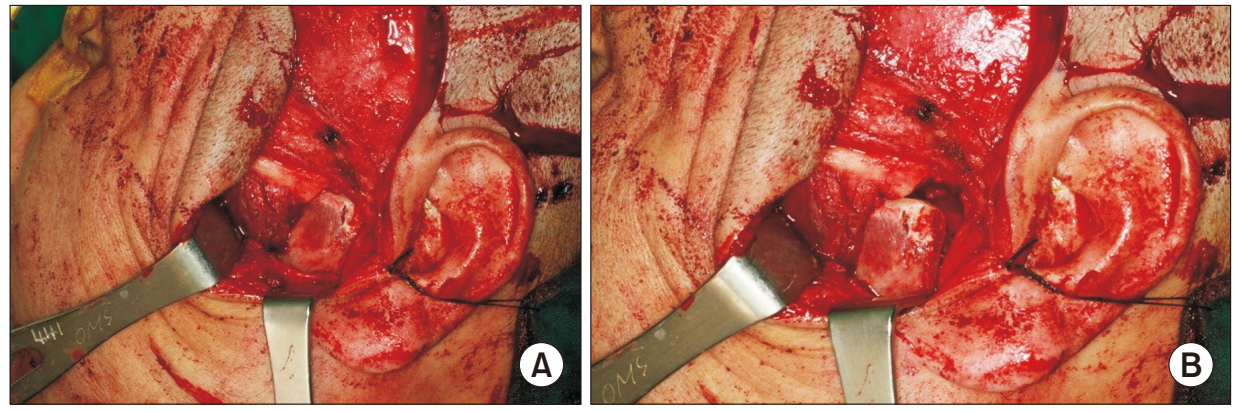

Fig. 2. A. Trimmed end portion of the costochondral graft loosely contacted the temporomandibular joint in the centric occlusion state. B. Trimmed end portion of the costochondral graft moved along the articular fossa as mouth opening increased.

Moon Gi Choi: Temporomandibular joint reconstruction with costochondral graft: case series study. $J$ Korean Assoc Oral Maxillofac Surg 2021
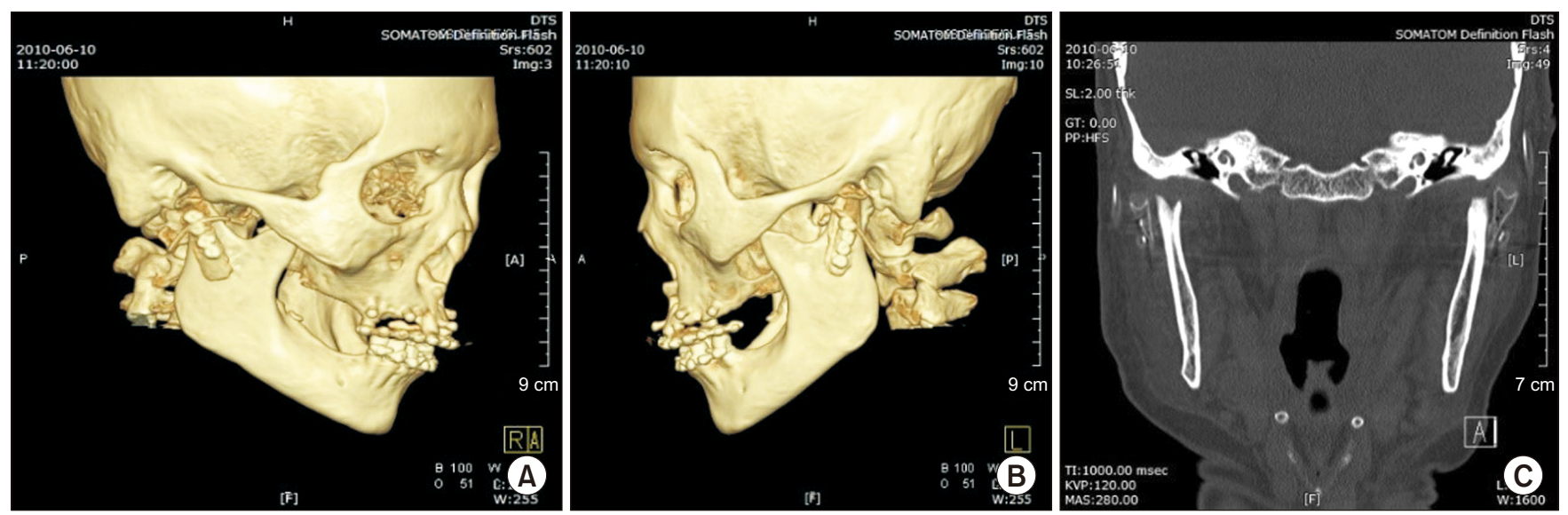

Fig. 3. A, B. On three-dimensional computed tomography, the costochondral graft was fixed in a good position. C. On coronal plane view, costochondral grafts were positioned on the lateral surface of the articular fossa.

Moon Gi Choi: Temporomandibular joint reconstruction with costochondral graft: case series study. J Korean Assoc Oral Maxillofac Surg 2021 


\section{Case 1}

A 48-year-old female patient presented with bilateral condylar fractures due to a fall. Both fractures were within the capsule (level II fractures).(Fig. 1)

Initial mouth opening was limited due to pain. She was partially edentulous in both jaws and was wearing a partial denture, which was broken during the fall. She showed loss of vertical dimension. Because the fractured fragments were too small to fix, we planned their removal and bilateral condylar reconstruction using costochondral grafts. Temporary denture was fabricated for proper occlusion and vertical dimension. Two costochondral grafts were harvested from the 5th and 7th rib bones of the right chest through inframammary incision. The cartilage portion was trimmed to 2-3 $\mathrm{mm}$ in thickness. Both TMJs were approached through the preauricular route. Both TMJ discs were intact without tearing or perforation. Each graft was pushed upward to contact the disc slightly and fixed with the washer technique with a temporary denture. Condylar movement and occlusion were measured on mouth closing and at open positions.(Fig. 2)

Intermaxillary fixation for bony consolidation was used for three weeks following surgery, and active opening exercises were prescribed for the three months after that. On threedimensional computed tomography (CT), grafts were contacting a lateral surface of the articular fossa.(Fig. 3)

Shortly after operation, the patient complained of an uncomfortable itching sensation at the donor site stimulated by the bra strap.

The patient was followed for 10 years. At last examination, she was free of TMJ pain and donor site complications and showed near normal mandibular excursion and protrusion. Maximum mouth opening was $40 \mathrm{~mm}$, right and left excur-

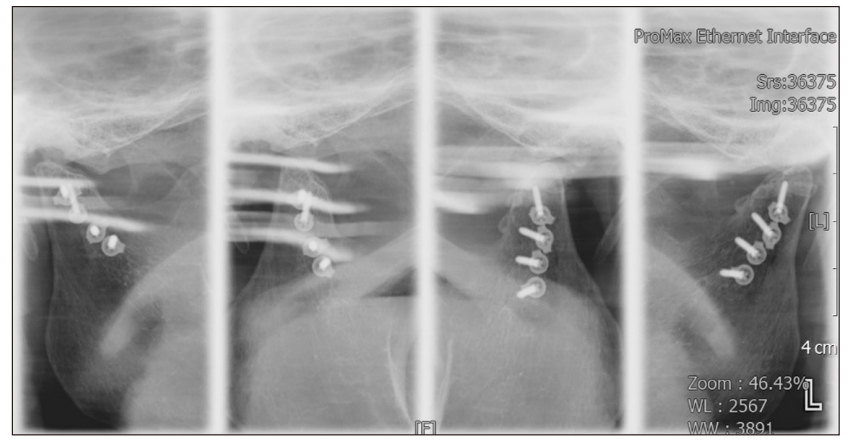

Fig. 4. At the last visit, the patient showed near normal mandibular excursion and protrusion.

Moon Gi Choi: Temporomandibular joint reconstruction with costochondral graft: case series study. J Korean Assoc Oral Maxillofac Surg 2021 sion was about $7 \mathrm{~mm}$, and protrusive movement was about 6 mm.(Fig. 4) There was no facial asymmetry. Though Frey's syndrome had developed, she refused treatment. She showed no sensory nerve damage around the TMJ.

\section{Case 2}

A 48-year-old male patient visited the emergency room due to painful swelling on the right hemiface. On CT, there was abscess formation and bony destruction around the right condyle and ramus area.(Fig. 5) The patient was diagnosed with submasticatory space abscess and acute suppurative osteomyelitis on the right condyle and ramus.

At first operation, incision and drainage and surgical debridement were performed through a submandibular approach. After three weeks, the infection was controlled. A second operation was performed for condyle and ramus reconstruction with a right 6 th rib costochondral graft.

Submandibular incision was used without preauricular approach. The disc of the TMJ was degenerated and removed. Costal cartilage was trimmed to $2-3 \mathrm{~mm}$ in thickness, and the graft was fixed with plate and screw fixation and wiring.

Intermaxillary fixation was used for three weeks after surgery. After releasing the intermaxillary fixation, active mouth opening exercise with therabite was performed for three months. The patient was followed up for six months, at which maximum mouth opening was $35 \mathrm{~mm}$, protrusion was $7 \mathrm{~mm}$,

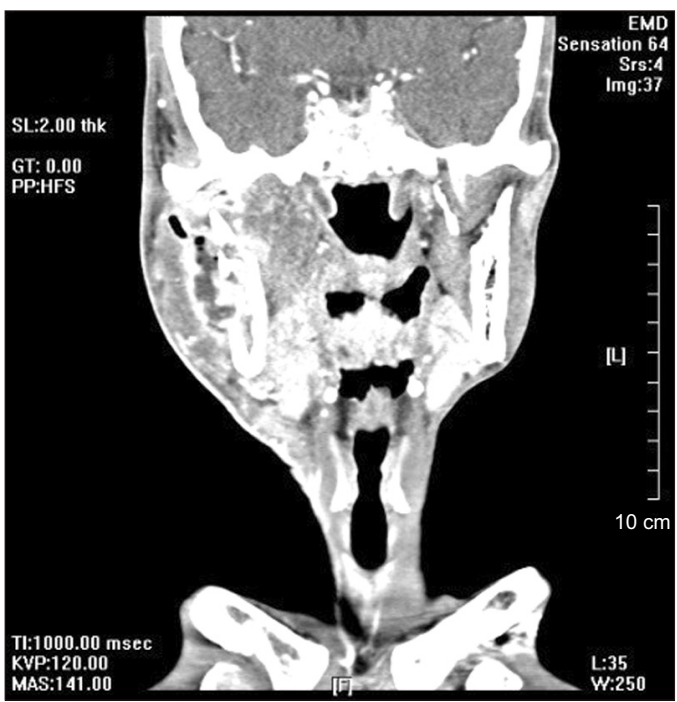

Fig. 5. On computed tomography, submasticatory space abscess and bony destruction were observed around the condyle and ramus.

Moon Gi Choi: Temporomandibular joint reconstruction with costochondral graft: case series study. J Korean Assoc Oral Maxillofac Surg 2021 
left excursion was $1 \mathrm{~mm}$, and right excursion was $7 \mathrm{~mm}$.

On opening, the mandible deviated to the right side due to limited movement of the right neo-condyle, but centric occlusion was obtained.

The patient was free of pain and donor site complications. He died of unrelated vascular accident eight months after operation.

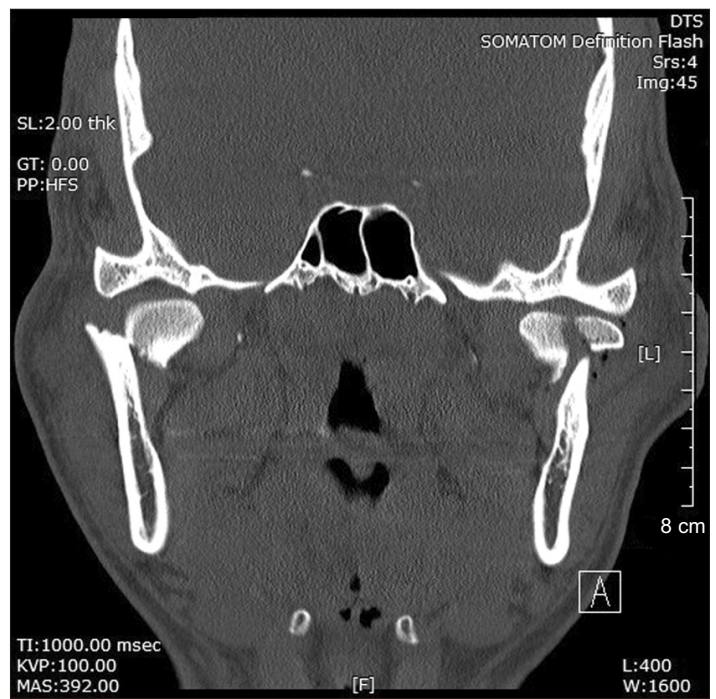

Fig. 6. On computed tomography, right and left condylar fractures were level 2, but there were multiple fragments on the left side. Moon Gi Choi: Temporomandibular joint reconstruction with costochondral graft: case series study. J Korean Assoc Oral Maxillofac Surg 2021

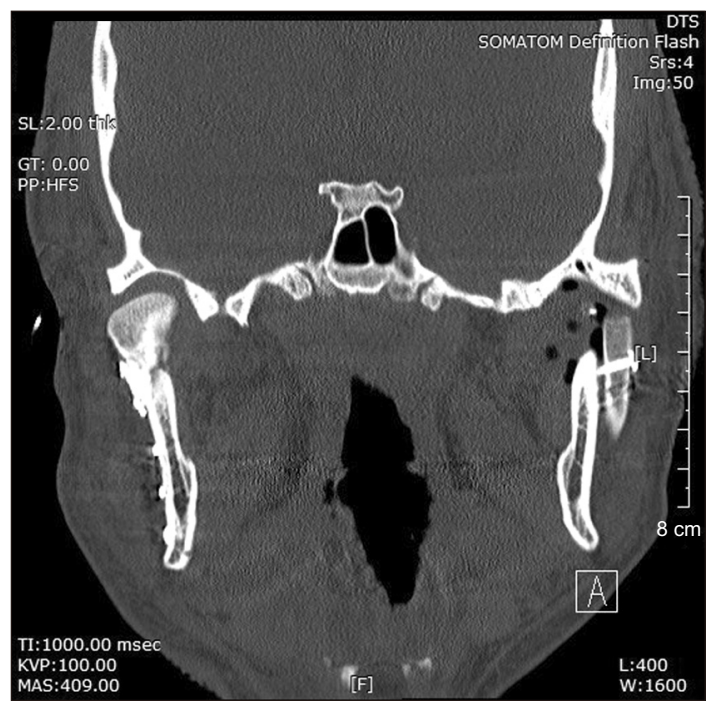

Fig. 7. The right condyle was fixed openly, and the left condyle was reconstructed with a costochondral graft. The cartilage portion of the graft was positioned well into the condylar fossa.

Moon Gi Choi: Temporomandibular joint reconstruction with costochondral graft: case series study. J Korean Assoc Oral Maxillofac Surg 2021

\section{Case 3}

A 17-year-old male patient suffered a facial bone fracture in a car accident. The diagnosis was bilateral intracapsular condylar fractures and symphysis fracture.(Fig. 6)

The right condyle was intact enough to be repaired, but the left condyle was fractured into multiple pieces. Our plan was open reduction and fixation of the right condyle and symphysis and reconstruction with a costochondral graft for the left

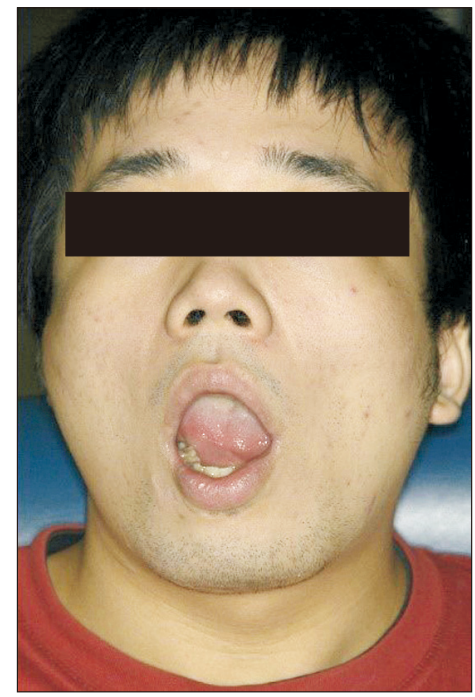

Fig. 8. Patient showed limited mandibular excursion, but maximum mouth opening was $45 \mathrm{~mm}$ in length.

Moon Gi Choi: Temporomandibular joint reconstruction with costochondral graft: case series study. J Korean Assoc Oral Maxillofac Surg 2021

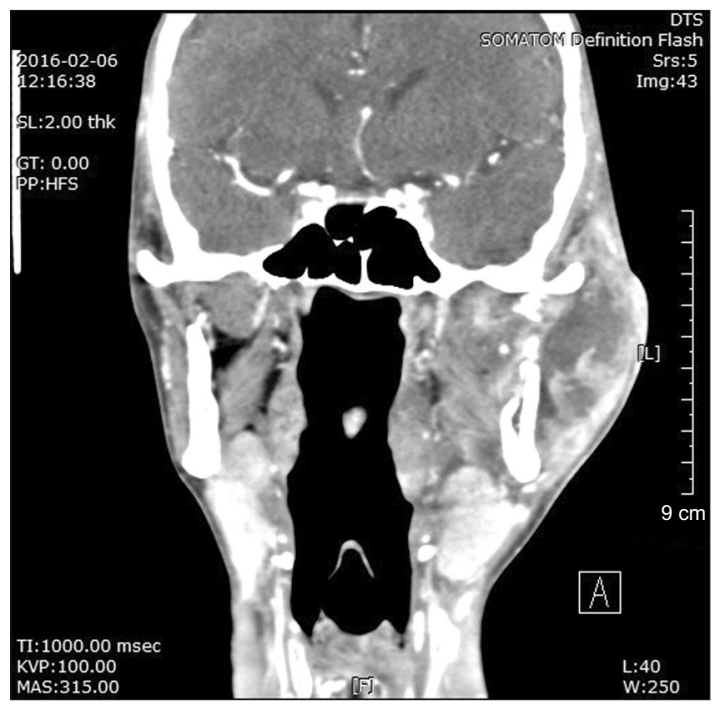

Fig. 9. On computed tomography, submasticatory space abscess and bony destruction were observed around the condyle and ramus.

Moon Gi Choi: Temporomandibular joint reconstruction with costochondral graft: case series study. J Korean Assoc Oral Maxillofac Surg 2021 
condyle. A $5 \mathrm{~cm}$ length of costochondral graft was harvested from the right chest, and cartilage was trimmed to $2-3 \mathrm{~mm}$ in thickness. Surgical approach was both preauricular and submandibular. The graft was fixed with a plate and screws using a washer technique.(Fig. 7)

The patient was followed up for 4 years 3 months. Maximum mouth opening was $45 \mathrm{~mm}$ with $5 \mathrm{~mm}$ left deviation, protrusion was $4 \mathrm{~mm}$ with $2 \mathrm{~mm}$ left deviation, left excursion was $2 \mathrm{~mm}$, and right excursion was $4 \mathrm{~mm}$.(Fig. 8)

On opening, the mandible deviated to the right side, but centric occlusion was obtained. The patient complained of intermittent pain and swelling on the left TMJ. An antiinflammatory drug reduced these problems.

\section{Case 4}

A 48-year-old male patient visited the emergency room due to painful swelling on the left hemiface. On CT, there was an abscess formation in the submasticatory space and bony destruction around the left condyle and ramus area.(Fig. 9) The patient was diagnosed with submasticatory space abscess and acute suppurative osteomyelitis of the left condyle and ramus. The patient was partially edentulous.

After infection was controlled, the left condyle and ramus were to be reconstructed with a costochondral graft after removal of the destroyed bony mass. To maintain the vertical dimension, upper and lower partial dentures were fabricated on an articulator. The surgical approach was submandibular and extended preauricular. Despite severe infection, the disc was intact. The washer technique was used for graft fixation. During the operation, neocondylar movement was good.

The patient was followed up for two months. Maximum mouth opening was $50 \mathrm{~mm}$ without deviation, and protrusion was $4 \mathrm{~mm}$ without deviation. Left excursion was $1 \mathrm{~mm}$, and right excursion was $5 \mathrm{~mm}$.

The vertical dimension was maintained without a denture. The patient was free of pain but produced audible TMJ sound. Donor site complication was negligible. After extrac- tion of all remaining teeth, complete dentures were installed.

The four cases are summarized in Table 1.

\section{Discussion}

Through reconstruction of the TMJ, we can restore the form and function of the mandible, reduce disability and pain, and prevent progression of disease.

The most widely accepted autogenous technique is costochondral graft. Costochondral transplantation has many advantages over other autogenous transplants. Harvesting is easy and has minimal impact on patients. The graft bears functional loading well and show excellent biocompatibility because the costal cartilaginous cap is composed of hyaline cartilage $e^{1,4}$. A costochondral graft has potential for growth in children. Despite these advantages, there are many potential problems including fracture of the graft, further ankylosis, poor rib bone quality, possibility of separation of cartilage from rib bone, variable growth behavior of the graft, and donor-site morbidity ${ }^{2,3}$. Although donor site morbidity such as pleural tear, pneumothorax, pleural effusion, atelectasis, and emphysema are minimal, it can be bothersome to the patients ${ }^{1-4}$.

Because ribs from the ipsilateral side do not match well to the angulation of the neocondyle, the contralateral rib is preferred for joint reconstruction. The 5th, 6th, and 7 th ribs are commonly used ${ }^{1,3}$. Irrespective of the case, the rib tissue was harvested from the right thoracic cage to distinguish postoperative pain from cardiac pain.

When a vascularized free rib graft is needed, it is collected from the fourth ib $^{5}$. Reconstruction of the infratemporal fossa and condyle using vascularized costochondral and calvarial bone grafts was reported by Jang et al. ${ }^{6}$. When wide reconstruction including the condyle is not indicated, nonvascularized costochondral graft can be sufficient.

If the rib is harvested from the ipsilateral side, it requires additional contouring. Because rib bone has flexibility, it can be bent according to a contoured plate. When angulation of the rib bone is irregular and hard to modify, the superolateral

Table 1. Summary of the four cases

\begin{tabular}{|c|c|c|c|c|c|c|c|c|}
\hline $\begin{array}{l}\text { Case } \\
\text { No. }\end{array}$ & Etiology & $\begin{array}{c}\mathrm{F} / \mathrm{U} \\
\text { period }\end{array}$ & $\begin{array}{l}\text { Operation } \\
\text { site }\end{array}$ & $\begin{array}{c}\text { Maximum } \\
\text { opening }(\mathrm{mm})\end{array}$ & $\begin{array}{l}\text { Rt. excursion } \\
(\mathrm{mm})\end{array}$ & $\begin{array}{l}\text { Lt. excursion } \\
(\mathrm{mm})\end{array}$ & $\begin{array}{l}\text { Protrusion } \\
(\mathrm{mm})\end{array}$ & Complications \\
\hline 1 & Trauma & $10 \mathrm{yr}$ & Both & 40 & 6 & 7 & 6 & Frey's syndrome \\
\hline 2 & Osteomyelitis & $6 \mathrm{mo}$ & Rt. & 35 & 7 & 1 & 7 & No \\
\hline 3 & Trauma & $4.5 \mathrm{yr}$ & Lt. & 45 & 4 & 2 & 4 & $\begin{array}{l}\text { Intermittent pain } \\
\text { \& swelling }\end{array}$ \\
\hline 4 & Osteomyelitis & $2 \mathrm{mo}$ & Lt. & 50 & 5 & 1 & 4 & TMJ sound \\
\hline
\end{tabular}

(F/U: follow-up, Rt.: right, Lt.: left, TMJ: temporomandibular joint)

Moon Gi Choi: Temporomandibular joint reconstruction with costochondral graft: case series study. J Korean Assoc Oral Maxillofac Surg 2021 
surface of the ramal bone can be trimmed so that the end of the rib bone is not displaced from the glenoid fossa ${ }^{4}$. The rib is harvested through an inframammary incision at the level of the end of the pectoralis major muscle, approximately $2.5 \mathrm{~cm}$ below the nipple ${ }^{1,7}$. The female patient in this case series (case No. 1) complained of itching sensation at the incision site due to stimulation caused by a brassiere strap. This suggests that such an incision location should be avoided.

If two ribs are needed, they should not be harvested from both thoracic cages, which involves a complicated procedure. Instead, grafts were harvested from one side only. If two grafts were harvested for bicondylar reconstruction, tissue was collected from separate rib bones to prevent sinking of the thoracic cage, leaving intact rib bone between them. A graft size of approximately $1 \mathrm{~cm}$ of cartilage and 3 to $4 \mathrm{~cm}$ of bone is typical.

A surgical knife is used to trim the costal cartilage so that it imitates the condylar head and fits in the articular fossa as accurately as possible ${ }^{4}$. The costal cartilage cap at the end of the rib bone is trimmed to a cartilage length from 0.4 to 2 $\mathrm{cm}$. Most surgeons prefer a smaller cartilage cap to prevent separation of cartilage from the rib bone at the costochondral junction and to reduce the chance of graft overgrowth ${ }^{8}$. We used a cartilage cap of only 2 to $3 \mathrm{~mm}$ in height.

Intermaxillary fixation to allow initial consolidation over a period of three weeks can be followed by a soft diet for another three weeks ${ }^{9}$. This situation is likely to prevent early mobilization and dysfunction of the mandible. We used intermaxillary fixation after costochondral graft in the first two cases, but subsequently determined that proper fixation does not require intermaxillary fixation. When the graft was fixed, excessive tightening of the screw could cause fracture of the rib bone longitudinally, and the screw would dig into the rib bone, hindering rigid fixation. To obtain a strong fix to weak ribs, the "washer" technique has been developed. The hole of the plate disperses pressure of the head of the screw when tightening $^{4,9-11}$. The surgeon has the option to cut the plate into a washer and use one hole. Because the surgeon must bend the plate accurately within a narrow surgical field, using a washer to fix the graft to the mandible is easier than using a plate. We used this washer technique in all instances.

A combination of endaural or preauricular incision and posterior submandibular incision is necessary to properly place and secure the rib graft ${ }^{4}$. We used both preauricular and submandibular approaches in the first two cases, but subsequently used only one approach in the following cases.

Several authors suggest that interpositional grafts such as dermal or temporomyofascial be used in conjunction with costochondral graft ${ }^{4}$. However, when the rib has cartilage on its end, interpositional grafts are not necessary ${ }^{5}$.

Compared to costochondral graft, other autogenous grafts have their own advantages and shortcomings. The sternoclavicular joint is similar to the TMJ anatomically and physiologically. It also has a cartilaginous cap and growth potential. However, harvest of the sternoclavicular joint might leave a conspicuous scar and cause donor-site morbidity through damage to the great vessels and eventual shoulder instability $^{2.3}$. Metatarsophalangeal joint graft has articular cartilage and growth potential, with similar shape and size to the TMJ. Though such anatomical fit is possible, microvascular anastomosis is necessary, and loss of a toe poses functional and aesthetic deficits ${ }^{2,3}$. An iliac graft contains a large amount of bone and is preferred with large mandibular reconstruction. Harvest of the iliac crest can cause greater morbidity than harvesting of a costochondral graft and can involve prolonged postoperative pain, lateral femoral nerve damage, fracture of the anterior spine of the ilium, and abdominal herniation ${ }^{2,3}$. Such iliac grafts for condylar reconstruction must be carried out in select cases ${ }^{12}$. The fibula is narrow and easily fit to the glenoid fossa. However, this graft technique requires microvascular anastomosis, and the fibular bone does not have cartilage. Donor-site morbidity is serious and long lasting. Big toe flexion contractures and valgus deformity can result. Large mandibular defect is first choice for fibular flap ${ }^{2,3}$.

A number of alloplastic implants has been introduced for reconstruction of the TMJ to avoid problems of autogenous grafts. A better shaped neocondyle can be produced in the factory. Successful alloplastic prosthesis would offer several advantages. Without harvest of autogenous tissue, there is no donor site morbidity and operation time decreases. Immediate reconstruction becomes possible. Also, active physiotherapy can be performed without delay. Autogenous grafts have unpredictable resorption, but lack of absorption in an alloplastic implant produces stable occlusion. This technique does not require intermaxillary fixation, and immediate jaw exercise is possible ${ }^{1,13}$. In our cases, resorption was detected on radiology, but severe complications did not arise.

The potential disadvantages of alloplastic implants are related to wearing, loosening, and lack of growth. Wear particles are a source of foreign body reaction. Loosening of the implant results in changes of occlusion and sometimes leads to implant fracture ${ }^{1}$. High cost and heterotopic bone formation around the implant are additional problems ${ }^{1}$. With any total joint alloplastic system, the patient only has rotational movement because the lateral pterygoid muscle does not in- 
sert onto the condyle. Only a $32-35 \mathrm{~mm}$ intraincisal opening can be expected ${ }^{4}$.

All the above cases were treated with autogenous tissue costochondral grafts because insurance companies did not cover TMJ alloplastic systems at that time and the equipment was too expensive.

Although the alloplastic implant is now an accepted treatment and has produced successful results, autogenous reconstruction of TMJ remains one of the treatments of choice. If the costochondral graft takes well, revisional surgery that is sometimes required for alloplastic implants is obviated. Costochondral grafts are always preferred for patients with growth potential because alloplastic implants do not provide for growth of the condyle. Although of limited dimension, costochondral grafts provide better mandibular movements. Resulting mouth opening is excellent, and excursion is limited, neither of which can be provided by alloplastic joints.

In the case 1 bilateral costochondral graft, mandibular movement was excellent, the patient was free of pain, and there was no re-ankylosis caused by ectopic bone formation. The other cases also showed good mandibular movement with limited excursion and were free of pain. No cases showed facial asymmetry or ankyloses.

Although the definitive prognosis is yet to be determined, we think that a costochondral graft can be used with minimal complications and acceptable results for autogenous condylar reconstruction.

\section{Author's Contributions}

The data collection and manuscript was performed by M.G.C.

\section{Acknowledgements}

This paper was supported by Wonkwang University in 2019.

\section{Consent for Publishing Photographs}

Written informed consent was obtained from the patient for publication of this article and accompanying images.

\section{Conflict of Interest}

No potential conflict of interest relevant to this article was reported.

\section{References}

1. Saeed N, Hensher R, McLeod N, Kent J. Reconstruction of the temporomandibular joint autogenous compared with alloplastic. Br J Oral Maxillofac Surg 2002;40:296-9. https://doi.org/10.1016/ s0266-4356(02)00139-0

2. Khadka A, Hu J. Autogenous grafts for condylar reconstruction in treatment of TMJ ankylosis: current concepts and considerations for the future. Int J Oral Maxillofac Surg 2012;41:94-102. https:// doi.org/10.1016/j.ijom.2011.10.018

3. Mohan MC, Prasad BR, Bhat S, Bhat SS. Reconstruction of condyle following surgical correction of temporomandibular joint ankylosis: current concepts and considerations for the future. NUJHS 2014;4:39-46.

4. Quinn PD. Color atlas of temporomandibular joint surgery. St. Louis: Mosby; 1998.

5. Bayat M, Badri A, Moharamnejad N. Treatment of temporomandibular joint ankylosis: gap and interpositional arthroplasty with temporalis muscle flap. Oral Maxillofac Surg 2009;13:207-12. https://doi.org/10.1007/s10006-009-0174-4

6. Jang HW, Kim NK, Lee WS, Kim HJ, Cha IH, Nam W. Mandibular condyle and infratemporal fossa reconstruction using vascularized costochondral and calvarial bone grafts. J Korean Assoc Oral Maxillofac Surg 2014:40:83-6. https://doi.org/10.5125/jkaoms.2014.40.2.83

7. Fernandes R, Fattahi T, Steinberg B. Costochondral rib grafts in mandibular reconstruction. Atlas Oral Maxillofac Surg Clin North Am 2006;14:179-83. https://doi.org/10.1016/j.cxom.2006.05.007

8. Chen CT, Lai JP, Chen YR. Costochondral graft in acute mandibular condylar fracture. Plast Reconstr Surg 1997;100:1234-9. https:// doi.org/10.1097/00006534-199710000-00024

9. Mosby EL, Hiatt WR. A technique of fixation of costochondral grafts for reconstruction of the temporomandibular joint. J Oral Maxillofac Surg 1989;47:209-11. https://doi.org/10.1016/s02782391(89)80122-3

10. Shetty KP, Mehta R, Mokal N. An innovative technique for fixing costochondral grafts. Plast Reconstr Surg 2000;106:1658-9. https:// doi.org/10.1097/00006534-200012000-00048

11. Kaban LB, Troulis MJ. Pediatric oral and maxillofacial surgery. Philadelphia: W.B. Saunders; 2004:351.

12. Matukas VJ, Szymela VF, Schmidt JF. Surgical treatment of bony ankylosis in a child using a composite cartilage-bone iliac crest graft. J Oral Surg 1980;38:903-5.

13. Kummoona R. Chondro-osseous iliac crest graft for one stage reconstruction of the ankylosed TMJ in children. J Maxillofac Surg 1986;14:215-20. https://doi.org/10.1016/s0301-0503(86)80292-2

How to cite this article: Choi MG. Temporomandibular joint reconstruction with costochondral graft: case series study. J Korean Assoc Oral Maxillofac Surg 2021;47:128-134. https://doi. org/10.5125/jkaoms.2021.47.2.128 